\title{
CONSTRUINDO POSSIBILIDADES EM BOURDIEU PARA ANÁLISE DO CAMPO DA ENFERMAGEM
}

Tarciso Feijó da Silva1 , Helena Maria Scherlowski Leal David², Fabiana Ferreira Koopmans²,Donizete Vago Daher ${ }^{3}$

Objetivo: compreender e estabelecer relação de sentido entre os conceitos fundamentais de Pierre Bourdieu e o campo da Enfermagem. Metodologia: estudo de natureza reflexiva realizado a partir do conteúdo teórico utilizado na disciplina de Pierre Bourdieu e a produção social da cultura, do conhecimento e da informação, do curso de doutorado do Programa de Pós-Graduação em Enfermagem da Universidade do Estado do Rio de Janeiro e do II Seminário "Pierre Bourdieu e a produção social da cultura, do conhecimento e da informação". Resultados: O campo da Enfermagem foi identificado como um espaço onde são construídos saberes e desenvolvidas práticas em torno dos objetos que justificam sua existência. O lugar ocupado pelos profissionais neste campo, por sua vez, mostrou-se dependente da aquisição de capital e de sua acumulação por parte dos profissionais inseridos no mesmo. Conclusão: os conceitos de habitus, campo social, capital social e cultural permitiram reconstruir sentidos sobre o campo da Enfermagem produzindo reflexão sobre a relevância do mesmo para a área das ciências da saúde.

Descritores: Enfermagem, ciências da saúde, conhecimento.

\section{BUILDING POSSIBILITIES IN BOURDIEU FOR ANALYSIS OF THE FIELD OF NURSING}

Objective: to understand and establish a relation of meaning between the fundamental concepts of Pierre Bourdieu and the field of Nursing. Methodology: a reflexive study based on the theoretical content used in the discipline of Pierre Bourdieu and the social production of culture, knowledge and information, the doctorate course of the Postgraduate Program in Nursing of the State University of Rio de January and the II Seminar "Pierre Bourdieu and the social production of culture, knowledge and information". Results: The field of Nursing was identified as a space where knowledge is developed and practices developed around the objects that justify its existence. The place occupied by professionals in this field, in turn, was dependent on the acquisition of capital and its accumulation by the professionals inserted in it. Conclusion: the concepts of habitus, social field, social and cultural capital allowed us to reconstruct meanings about the field of Nursing, producing reflection on its relevance to the area of health sciences.

Descriptors: Nursing, healths ciences, knowledge

\section{CONSTRUYENDO POSIBILIDADES EN BOURDIEU PARAANÁLISIS DEL CAMPO DE LA ENFERMERÍA}

Objetivo: comprender y establecer relación de sentido entre los conceptos fundamentales de Pierre Bourdieu y el campo de la enfermería. Metodología: estudio de naturaleza reflexiva realizado a partir del contenido teórico utilizado en la disciplina de Pierre Bourdieu y la producción social de la cultura, del conocimiento y de la información, del curso de doctorado del Programa de Postgrado en Enfermería de la Universidad del Estado de Río de Janeiro Enero y del II Seminario "Pierre Bourdieu y la producción social de la cultura, del conocimiento y de la información". Resultados: El campo de la Enfermería fue identificado como un espacio donde se construyen saberes y desarrolladas prácticas en torno a los objetos que justifican su existencia. El lugar ocupado por los profesionales en este campo, a su vez, se mostró dependiente de la adquisición de capital y de su acumulación por parte de los profesionales insertados en el mismo. Conclusión: los conceptos de habitus, campo social, capital social y cultural permitieron reconstruir sentidos sobre el campo de la Enfermería produciendo reflexión sobre la relevancia del mismo para el área de las ciencias de la salud.

Descriptores: Enfermería, ciencias de la salud, conocimiento.

'Universidade Estácio de Sá-RJ- Email: tarcisofeijo@yahoo.com.br

2Universidade do Estado do Rio de Janeiro-UERJ.

${ }^{3}$ Universidade Federal Fluminense-UFF/RJ. 


\section{INTRODUÇÃO}

Esta reflexão partiu das discussões na disciplina do Curso do Doutorado sobre Pierre Bourdieu e do II Seminário "Pierre Bourdieu e a produção social da cultura, do conhecimento e da informação", ambos relacionados ao Programa de Pósgraduação em Enfermagem da Faculdade de Enfermagem da Universidade do Estado do Rio de Janeiro (PPGENF/UERJ). Os diferentes conceitos apresentados pela disciplina, a partir do referencial teórico de Pierre Bourdieu, contribuíram para a construção de sentidos no que tange a Enfermagem, "abrindo" a cortina para as seguintes indagações: Qual relação existente entre os conceitos de Pierre Bourdieu e o campo da Enfermagem? De que forma estes conceitos podem contribuir para uma análise crítica deste campo?

Para compreender de que forma os conceitos do autor articulam-se com a Enfermagem foi necessário primeiramente entender que a Enfermagem como campo do conhecimento está vinculada a área das ciências da saúde e subdividida em subáreas: Enfermagem médico-cirúrgica, obstétrica, pediátrica, de doenças psiquiátricas e de saúde públical. O enfermeiro a partir de competências relacionadas à sua formação primária na graduação e complementar nos diferentes cursos de especialização existentes, assim como em programas de mestrado e doutorado, tem adquirido autonomia para tomada de decisão no exercício da profissão na assistência e na docência. Existem, no entanto, normativas e políticas institucionais que regulam a autonomia do profissional enfermeiro e contribui para manter cada subárea da Enfermagem permeável àqueles que possuem qualificação e conhecimento. Esta prerrogativa é importante considerando que, o que cada indivíduo constrói como produto do processamento, da interpretação e da compreensão de uma informação contribui para o fazer ciência ${ }^{2}$ e que existe uma pluralidade de significados atrelados a este termo, podendo ser entendida como um acervo de conhecimentos desenvolvido, acumulado, transformado e reestruturado de acordo com a integração própria de cada campo ${ }^{3}$.

A Enfermagem, como um campo de saberes e práticas ao longo do século XX construiu, cumulativamente seu conhecimento e produziu historicamente suas práticas. $\mathrm{O}$ instrumental arquitetado para orientar a Enfermagem, na busca de sua autonomia, como campo específico de saber foram as teorias de Enfermagem e estas permitiram uma delimitação de seus limites de atuação, no trabalho com outros profissionais ${ }^{4}$. Apesar da ampliação do campo de desenvolvimento de trabalho e das novas oportunidades, ainda há várias discussões sobre o fazer no campo da Enfermagem ${ }^{5}$.

Neste contexto, a sociologia de Bourdieu e seus conceitos, geralmente articulados, são empregados em diversos campos de conhecimentob e podem ser utilizados para análise do campo da saúde e, por consequência, do campo da Enfermagem. Estes campos (saúde e especificamente Enfermagem) são articulados ao conjunto da sociedade e sofrem influências políticas, sociais e culturais no contexto do qual estão inseridos, transcendendo desta forma, os limites setoriais, na medida em que depende de políticas sociais, micro e macroeconômicas, relações comportamentais, ações institucionais e individuais ${ }^{7}$ para subsistir como campo e ciência e para legitimação da sua autonomia. Bourdieu procurou romper, no conjunto de suas obras, as fronteiras disciplinares e as divisões de áreas de conhecimento, contribuindo e influenciando outros diferentes campos8.

Nesta perspectiva, o objetivo deste artigo é compreender e estabelecer relação de sentido entre os conceitos fundamentais de Pierre Bourdieu e o campo da Enfermagem.

\section{METODOLOGIA}

Trata-se de uma reflexão com fundamentação nos conceitos fundamentais de Pierre Bourdieu, tais como habitus, violência simbólica, campo social e capital e suas interfaces com o campo de conhecimento e práticas da Enfermagem. Esta discussão perpassou pela Disciplina de Pierre Bourdieu, do Curso do Doutorado e do II Seminário "Pierre Bourdieu e a produção social da cultura, do conhecimento e da informação", ambos relacionados ao Programa de Pós-graduação em Enfermagem da Faculdade de Enfermagem da Universidade do Estado do Rio de Janeiro (PPGENF/UERJ), ocorridos no decorrer do ano de 2016.

\section{O desvelar da Enfermagem à luz dos conceitos de Bourdieu}

Os estudos de Bourdieu permitiram identificar a Enfermagem como um subcampo do campo da saúde. Os subcampos (ou subsistemas) de acordo com o autor são regiões menores de um campo, os quais conservam a mesma dinâmica deste. Dessa forma, assim como nos demais campos e subcampos, os grupos detentores de maior volume de capital e melhor posição na estrutura deles, tendem a manterthes também o controle político?. Utilizaremos, no entanto, nesta discussão, o termo campo quando nos referirmos à Enfermagem orientados pela Classificação das áreas de conhecimento do Conselho Nacional de Desenvolvimento Científico e Tecnológico $(\mathrm{CNPq})^{1}$. A noção de campo e subcampo apresentada pelo autor abre espaço para discutir relações de domínio existentes de acordo com o capital dos agentes inseridos nos mesmos. Tomada de empréstimo das investigações econômicas do Marxismo, a noção de capital empregada por Bourdieu auxilia no entendimento da posição que o indivíduo ocupa no campo, e segundo ele os detentores de maior capital são tidos como dominantes e o 
de menor capital como dominados. Os dominados, por sua vez, enfrentam o que autor chamou de violência simbólica, que está atrelada ao acesso às oportunidades de adquirir capital e a imposição do capital dos dominantes (cultural, social, político, econômico, linguístico, profissional) ${ }^{10}$.

O conceito de capital para o campo da Enfermagem (ou subcampo de acordo com Bourdieu) é potente na medida em que permite compreender a luta de classes existentes entre este campo e os outros campos da área da saúde. Possibilita também analisar sob a vertente de dominantes e dominados as lutas existentes pelo poder entre os profissionais da mesma categoria no próprio campo da Enfermagem. É importante entender que os agentes (enfermeiros) possuem em comum um conjunto de interesses essenciais, isto é, "[...] tudo o que está ligado à existência do campo e isto leva à construção de uma cumplicidade objetiva que permanece subjacente a todos os conflitos e antagonismos existentes".11:51 Porém, essa conivência, não se expressa formalmente, mas de forma tácita e consentida por todos os agentes (enfermeiros). Assim, apesar das relações entre os agentes (enfermeiros), integrantes do campo da Enfermagem, serem permeadas de cooperação, nelas coexistem o conflito e as disputas pela autoridade para falar e agir em nome do campol2. Alguns fatores contribuem para este processo, dentre eles a posição ocupada por cada agente no interior do campo, o capital que possuem e uso que fazem do mesmo, a relação de domínio existente no interior do campo e principalmente o que Bourdieu definiu como habitus.

O habitus seria aquilo que se adquire, mas que se encarna no corpo de forma durável sob a forma de disposições permanentes. Não deve assim, ser confundido com hábito que é considerado como ato repetitivo, mecânico, automático antes reprodutivo que produtivo ${ }^{13}$. As condições do exercício do habitus estão relacionadas a uma situação social, econômica, política - "estado particular dessa estrutura".10 Não é uma coleção passiva de conhecimentos, um conjunto de regras que aplicamos às situações sociais ${ }^{14}$. É o que está no ator, no sujeito, na linguagem, na biografia cultural e na história individual, conformada, essencialmente, pela sua trajetória social dentro da família e de seu sistema de valores (habitus primário) e pela herança cultural e formação educacional da escola (habitus secundário) ${ }^{10}$. No campo da Enfermagem a noção de habitus torna-se relevante por dois motivos, primeiro por ser um sistema de disposições orientado para a prática, ou seja, "[...] é um fundamento objetivo de condutas regulares, logo, da regularidade das condutas e, se é possivel prever as práticas [...] é porque o habitus faz com que os agentes que o possuem comportem-se de uma determinada maneira em determinadas circunstâncias"15:98 e segundo por ser considerado como uma matriz cultural que orienta os enfermeiros no campo da saúde, particularmente, no campo da Enfermagem ${ }^{12}$. O habitus, assim, indica a disposição incorporada, quase postural, disciplinando comportamentos, do que derivam campos estruturados e estruturantes ${ }^{10}$.

As necessidades incorporadas, convertidas em disposições geradoras de práticas e percepções fornecem sentido ao habitus, enquanto disposição geral e transponível. A estrutura estruturante que organiza as práticas e a percepção das práticas torna o habitus também uma estrutura estruturada, enquanto produtos estruturados (opus operatum) que a mesma estrutura estruturante (modus operandi) produz todas as práticas e obras do mesmo agente. Os esquemas geradores do habitus aplicam-se por simples transferência, aos mais diferentes domínios da prática8.

No campo da Enfermagem ou em outro campo da área das ciências da saúde existe um universo no qual estão inseridos os agentes e instituições que produzem ou difundem a arte, a literatura ou a ciência que é definida por Bourdieu como campo social. ${ }^{16}$ Este, segundo o autor, é um mundo social como outro qualquer, funciona de modo mais ou menos autônomo em relação ao macrocosmo, se caracteriza por ser um campo de forças e de lutas e seu funcionamento, é dependente da existência de objetos em disputa e de pessoas dotadas de habitus $^{16}$. Compreender o significado de campo social permite reconhecer o conjunto de ações, representações e interações sociais que enfrentam os sujeitos6. Os campos sociais se caracterizam por espaços sociais, mais ou menos restritos, onde as ações individuais e coletivas se dão dentro de uma normatização, criada e transformada constantemente por essas próprias ações. Dialeticamente, esses espaços, ou estruturas, trazem em seu bojo uma dinâmica determinada e determinante, na mesma medida em que sofrem influências e, portanto, modificações-de seus atores.

O campo social da Enfermagem apresenta-se, assim, como um espaço onde são construidos saberes e desenvolvidas práticas em torno dos objetos que justificam sua existência ${ }^{12}$. Cada campo corresponde a um habitus (sistema de disposições incorporadas) próprio do campo e apenas quem tiver incorporado o habitus próprio do campo tem condição de jogar o jogo e de acreditar na importância desse jogo. Perceber a Enfermagem como campo, desta forma, implica reconhecê-la como uma estrutura estruturada regida por um arcabouço teórico, códigos específicos e diretrizes práticas (as regras do jogo). Nesta estrutura, estão legalmente instituídas, com competências e habilidades definidas, três categorias: os enfermeiros, com nivel de ensino superior; os técnicos e auxiliares de Enfermagem, com nível médio. O grau de complexidade dos conteúdos curriculares e a proporcionalidade de distribuição da carga horária entre teoria e prática, entre outros fatores, garantem distribuição 
desigual de capital entre os profissionais da Enfermagem afetando a dinâmica do campo social.

O espaço social é um campo de lutas onde os agentes elaboram estratégias que permitem manter ou melhorar sua posição social. Essas estratégias estão relacionadas com os diferentes tipos de capital. A partir desta compreensão, pressupomos, que o lugar ocupado pelo enfermeiro, técnico e auxiliar de Enfermagem vai depender da aquisição de capital e de sua acumulação por parte dos mesmos no interior do campo da Enfermagem.

Segundo Bourdieu existem diferentes tipos de capital: econômico, social, cultural e simbólico. Todos, de alguma forma, poderiam ser aplicados para análise do campo da Enfermagem, no entanto, os conceitos de capital social e capital cultural são abraçados nesta discussão por permitirem uma concepção mais abrangente. O capital social é a agregação de recursos atuais ou potenciais que tem ligação estreita com uma rede durável de relações institucionalizadas de reconhecimento e de inter-reconhecimento. As relações estabelecidas entre os indivíduos pertencentes a um determinado grupo não advêm apenas do compartilhamento de relações objetivas ou de proximidade no mesmo espaço econômico e social. Essas relações fundam-se também nas trocas materiais e simbólicas, cuja instauração e perpetuação supõem o reconhecimento dessa proximidade pelos agentes. Desta forma, o volume de capital social de um agente individual depende tanto da extensão da rede de relações que ele pode efetivamente mobilizar como do volume das diferentes formas de capital (econômico, cultural ou simbólico) que é propriedade exclusiva de cada um dos agentes a quem o indivíduo está ligado ${ }^{17}$.

Já o capital cultural refere-se "[...] ao conjunto das qualificações intelectuais produzidas pelo sistema escolar ou transmitidas pela familia"18:54 e existe sob três formas: no estado incorporado, ou seja, sob a forma de disposições duráveis do organismo; no estado objetivado, sob a forma de bens culturais - quadros, livros, dicionários, instrumentos, máquinas, que constituem indícios ou a realização de teorias ou de críticas dessas teorias, de problemáticas; e no estado institucionalizado, sob a forma de objetivação que é preciso colocar à parte porque, como se observa em relação ao certificado escolar, ela confere ao capital cultural - de que é, supostamente, a garantia - propriedades inteiramente originais17. A necessidade de capital social e cultural pelo profissional enfermeiro justifica-se, por dois motivos, o primeiro por identificar que o desafio para ocupação de espaços pela Enfermagem está relacionado, principalmente, à formação de enfermeiros competentes tecnicamente no cuidado humano, éticos, políticos e comprometidos com o papel social da Enfermagem; ${ }^{19}$ e o segundo por entender que é de grande relevância que os profissionais reflitam acerca do seu processo de formação, a partir de suas experiências e enfrentamentos no cotidiano de trabalho, confrontando as competências adquiridas e desenvolvidas no curso de graduação com a realidade profissional, sugerindo mudanças e contribuindo para uma formação capaz de atender as necessidades do exercício da profissão ${ }^{20}$.

\section{CONCLUSÃO}

A cortina que se abriu pela indagação inicial que motivou esta reflexão segue entreaberta entendendo não ter se esgotado as possibilidades de apreensão a partir dos conceitos de habitus, violência simbólica, campo social e capital social e cultural apresentados pela disciplina. Estes conceitos analisados amiúde na vasta obra de Bourdieu, outrora, podem revelar outras possibilidades, construir novos sentidos e serem úteis para a construção de novos conhecimentos que contribuam para análise da Enfermagem. Num primeiro momento procuramos apresentá-los estabelecendo a relação e a aplicabilidade dos mesmos com o campo da Enfermagem.

Desta forma, a partir da reflexão, a noção de habitus, campo social e capital emergiram como sendo relevantes para construção de sentidos sobre o campo da Enfermagem. O habitus por ser responsável por conformar a prática do profissional enfermeiro; o campo social por ditar normas que orientam a atuação dos profissionais no campo e pelo movimento que pressupõe pela ação dos atores que nele atuam; e ocapitalsocial e cultural porpermitirem compreender que os desafios afetos ao campo da Enfermagem estão relacionados à formação e a capacidade de análise crítica pelos profissionais da Enfermagem da relevância da profissão e do trabalho que desenvolvem.

Ademais, à luz de Bourdieu conjectura-se que as relações de domínio existentes no campo da Enfermagem entre os profissionais de nível médio e superior, assim como os mecanismos utilizados pelos que detêm maior capital social e cultural para manutenção das relações de domínio tendem a se constituir como elementos que contribuem para que este seja visto como importante campo da área das ciências da saúde. É relevante reiterar, no entanto, que no contexto da prática dos serviços de saúde enfermeiros dotados de habitus, capital social e cultural tendem cada vez mais a ocupar não só no campo da Enfermagem, mas também no campo da saúde papel de destaque, assumindo ações assistenciais e gerenciais. 


\section{REFERÊNCIAS}

1. Cnpq. Conselho Nacional de Desenvolvimento Cientifico e Tecnológico. Dados abertos: áreas de conhecimento. [Internet]. 2013. [cited 2017 Jul 23] Available from: http://www.cnpq.br/ web/guest/dados_abertos

2. Valente JA. Educação à distância no ensino superior: soluções e flexibilizações. Interface - Comunic., Saúde, Educ [Internet]. 2003. [cited 12 nov 2017]; 7 (12): 139-148. Available from: http:// www.scielo.br/pdf/icse/v7n12/v7n12a09.pdf

3. Schwartzman S. Um espaço para a ciência: a formação da comunidade cientifica no Brasil. Brasilia: Ministério de Ciência e Tecnologia, 2001. Available from: http://www.schwartzman.org. $\mathrm{br} / \mathrm{simon} / \mathrm{spacept/espaco.htm}$

4. Almeida MCP, Mishima SM, Pereira MJB, Palha PF, Villa TCS, Fortuna CM, Matumoto S. Enfermagem enquanto disciplina: que campo de conhecimento identifica a profissão? Rev. bras. enferm [Internet]. 2009. [cited 11 dez 2017]; 62 (5): 748-752. Available from: http://www.scielo.br/pdf/reben/v62n5/17.pdf

5. Lessa ABSL, Araujo CNV. A Enfermagem brasileira: reflexão sobre sua atuação política. Rev Min Enferm [Internet]. 2013. [cited 12 fev 2018]; 17(2): 481-487. Available from: http://www.reme. org.br/artigo/detalhes/664

6. Bourdieu P. Esboço de uma teoria da prática. In: Ortiz R, organizador. Bourdieu P. Sociologia. São Paulo: Ática, 1983.

7. Minayo MCS. O desafio do conhecimento: pesquisa qualitativa em saúde. 8a ed. São Paulo: Hucitec, 2004.

8.Bourdieu P. A Distinção: Crítica social do julgamento. Tradução Daniela Kern; Guilherme Teixeira. $2^{\underline{a}}$ ed. $2^{a}$ rev. Editora Zouk. Porto Alegre, 2015

9. Thiry-Cherques HR. Pierre Bourdieu: A teoria na prática. RAP [Internet]. 2006. [cited 07 jan 2018]; 40(1): 27-55. Available from: http://www.scielo.br/pdf/rap/v40nl/v40nla03.pdf

10. Nascimento DM, Marteleto R. A "informação construida" nos meandros da teoria social de Pierre Bourdieu. Datagrama zero - Rev. Ciência Info. [Internet]. 2004. [cited 19 fev 2018]; 5 (5): 1-9. Available from: http://www.brapci.inf.br/index.php/article/ download/7651
11. Domingos SM. Campo científico e interdisciplinaridade. In: Fernandes A, Guimarães FR, Brasileiro MCE. Org(s). O fio que une as pedras: a pesquisa interdisciplinar na pós-graduação. São Paulo: Biruta, 2002.

12. Lopes MEL, Sobrinho MD, Costa SFG. Contribuições da sociologia de Bourdieu para o estudo do subcampo da Enfermagem. Texto contexto - enferm [Internet]. 2013. [cited 10 ago 2018] 22 (3): 819-825. Available from: http://www.scielo.br/pdf/tce/ v22n3/v22n3a31.pdf

13. Bourdieu P. Questões de sociologia. Rio de Janeiro: Marco Zero, 1983.

14. Stevens G. O círculo privilegiado: fundamentos da distinção arquitetônica. Brasilia: Editora Universidade de Brasília, 2003.

15. Bourdieu P. Coisas ditas. São Paulo: Brasiliense, 2004.

16. Bourdieu P. Os usos sociais da ciência: por uma sociologia clinica do campo científico. São Paulo: UNESP, 2004

17. Bourdieu P. A dominação masculina. 5a ed. Rio de Janeiro: Bertrand Brasil, 2007.

18. Bonnewitz P. Primeiras lições sobre a sociologia de Pierre Bourdieu. Petrópolis: Vozes, 2005.

19. Corbellini VL, Santos BRL, Ojeda BS, Gerhart L M, Eidt OR, Stein SC, Mello DT. Nexos e desafios na formação profissional do enfermeiro. Rev Bras Enferm [Internet]. 2010. [cited 14 dez 2017]; 63(4): 555-60. Available from: http://www.scielo.br/pdf/reben/ v63n4/09.pdf

20. Meira MDD, Kurcgant P. Avaliação da formação de enfermeiros segundo a percepção de egressos. Acta Paul Enferm [Internet]. 2008. [cited 21 jan 2018]; 21(4): 556-61. Available from: http://www.scielo.br/pdf/ape/v2ln4/a04v2ln4.pdf 